\title{
SOME HELLY THEOREMS FOR MONOTONE FUNCTIONS
}

\author{
H. D. BRUNK, G. M. EWING AND W. R. UTZ ${ }^{1}$
}

1. Introduction. In this note we consider Helly theorems on the convergence of monotone functions of $n$ variables. Such theorems, first treated by E. Helly [3] in 1912 for $n=1$, occur frequently in one form or another (cf. [1, p. 389], [5, p. xii], [6, p. 27]) but the authors are unaware of an occurrence of the formulations and proofs as we give them here. These forms of the theorems are desirable in certain minimum problems under consideration by the authors.

To prove the Helly theorems we first find (Theorem 1) limitations on the set of discontinuities of monotone functions. This theorem in the case $n=2$ is due to W. H. Young [7] and a proof for $n=2$ is found in Hobson [4]. In proving the Young theorem, Hobson uses as a lemma a property of the limits of monotone functions which, as stated in Hobson, is false. ${ }^{2}$ This property does not play a role in the proof given here, (which was suggested by a proof found in Cramér [2, p. 79] for a similar theorem concerning distribution functions) but we correct the statement of the Hobson theorem in $\$ 3$ where we also determine limitations on the set of discontinuities of functions monotone in their separate variables and show that a Helly theorem is impossible for such functions.

2. Some Helly theorems. Throughout the paper $S$ shall denote the interval $a_{i} \leqq x_{i} \leqq b_{i}, i=1,2, \cdots, n$, of euclidean $n$-space. Consider a real valued function $F\left(x_{1}, x_{2}, \cdots, x_{n}\right)$ defined on $S$. For each $(n-2)$-tuple $p=\left(p_{1}, p_{2}, \cdots, p_{n-2}\right)$ and $1 \leqq \nu \leqq \eta \leqq n$ define

$F_{v \eta p}\left(x_{\nu}, x_{\eta} ; p\right)$

$$
=F\left(p_{1}, p_{2}, \cdots, p_{\nu-1}, x_{\nu}, p_{\nu}, \cdots, p_{\eta-2}, x_{\eta}, p_{\eta-1}, \cdots, p_{n-2}\right)
$$

provided

$$
\begin{array}{rrr}
a_{i} \leqq p_{i} \leqq b_{i}, & i=1,2, \cdots, \nu-1 ; \\
a_{i+1} \leqq p_{i} \leqq b_{i+1}, & i=\nu, \cdots, \eta-2 ; \\
a_{i+2} \leqq p_{i} \leqq b_{i+2}, & i=\eta-1, \cdots, n-2 .
\end{array}
$$

Presented to the Society, November 26,1955 ; received by the editors November 29, 1954 and, in revised form, September 30, 1955.

1 This research was supported by the United States Air Force through the Office of Scientific Research of the Air Research and Development Command.

${ }^{2}$ The referee has called our attention to the third edition of Hobson (1927) wherein this error is corrected. 
For each such $\nu, \eta, p$ and each pair $\alpha=\left(\alpha_{1}, \alpha_{2}\right), \beta=\left(\beta_{1}, \beta_{2}\right)$ with $a_{\nu} \leqq \alpha_{1} \leqq \beta_{1} \leqq b_{\nu}, a_{\eta} \leqq \alpha_{2} \leqq \beta_{2} \leqq b_{\eta}$ define

$$
\begin{aligned}
\Delta F_{\nu \eta p}(\alpha, \beta)= & F_{\nu \eta p}\left(\alpha_{1}, \alpha_{2} ; p\right)+F_{\nu \eta p}\left(\beta_{1}, \beta_{2} ; p\right) \\
& -F_{\nu \eta p}\left(\alpha_{1}, \beta_{2} ; p\right)-F_{\nu \eta p}\left(\beta_{1}, \alpha_{2} ; p\right) .
\end{aligned}
$$

In this note $F$ is said to be monotone if $F$ is monotone nondecreasing in $x_{i}$ for each $i=1,2, \cdots, n$, and if $\Delta F_{\nu \eta p}(\alpha, \beta) \geqq 0$ for all $\nu, \eta, \alpha, \beta, p$.

Thus a function is monotone provided all its first and second differences are non-negative. No higher differences than the second are needed in the paper. The requirement of non-negative differences is convenient in wording the proofs of the paper. However, the results hold if $F$ is of mixed monotoneity in its individual variables and one may also allow the inequality on the second difference to be $\Delta \geqq 0$ or $\Delta \leqq 0$ depending on $\nu, \eta$ in the quintuple $\nu, \eta, \alpha, \beta, p$ but for a fixed $\nu, \eta, \Delta \geqq 0$ or $\Delta \leqq 0$ for all admissible $\alpha, \beta, p$.

THEOREM 1. If $F\left(x_{1}, x_{2}, \cdots, x_{n}\right)$ is a real valued monotone function on $S$, then there is a set $R$ consisting of the points of a countable collection of $(n-1)$-spaces $x_{i}=\alpha_{i}^{j}, i=1,2, \cdots, n, j=1,2, \cdots$, such that $F$ is continuous at all points of $S-R$.

Proof. $F\left(b_{1}, b_{2}, \cdots, b_{i-1}, x_{i}, b_{i+1}, \cdots, b_{n}\right)$ is a monotone function of $x_{i}$; hence it has a countable number of discontinuities $x_{i}=\alpha_{i}^{j}$, $j=1,2, \cdots$. If we denote by $R$ the points of the sets $x_{i}=\alpha_{i}^{j}, i=1$, $2, \cdots, n, j=1,2, \cdots$ in $S$, then we shall show that $F$ is continuous at each point of $S-R$.

Suppose $\epsilon>0$ and $\left(x_{1}, x_{2}, \cdots, x_{n}\right) \in S-R$ are given. Let $\delta=\min \left(\delta_{1}, \delta_{2}, \cdots, \delta_{n}\right)$ where $\delta_{i}>0$ is such that

$$
\begin{aligned}
& F\left(b_{1}, \cdots, b_{i-1}, x_{i}+\left|h_{i}\right|, b_{i+1}, \cdots, b_{n}\right) \\
& \quad-F\left(b_{1}, \cdots, b_{i-1}, x_{i}-\left|h_{i}\right|, b_{i+1}, \cdots, b_{n}\right)<\epsilon / n \text { for }\left|h_{i}\right|<\delta_{i} .
\end{aligned}
$$

Consider $x+h=\left(x_{1}+h_{1}, x_{2}+h_{2}, \cdots, x_{n}+h_{n}\right)$ where $\left|h_{i}\right|<\delta_{i}, i=1$, $2, \cdots, n$. Then

$$
\begin{aligned}
&\left|F\left(x_{1}+h_{1}, \cdots, x_{n}+h_{n}\right)-F\left(x_{1}, \cdots, x_{n}\right)\right| \\
& \leqq F\left(x_{1}+\left|h_{1}\right|, \cdots, x_{n}+\left|h_{n}\right|\right)-F\left(x_{1}-\left|h_{1}\right|, \cdots, x_{n}-\left|h_{n}\right|\right) \\
&= \sum_{i=1}^{n}\left\{F\left(x_{1}+\left|h_{1}\right|, \cdots, x_{i}+\left|h_{i}\right|, x_{i+1}-\left|h_{i+1}\right|, \cdots, x_{n}-\left|h_{n}\right|\right)\right. \\
&\left.\quad-F\left(x_{1}+\left|h_{1}\right|, \cdots, x_{i-1}+\left|h_{i-1}\right|, x_{i}-\left|h_{i}\right|, \cdots, x_{n}-\left|h_{n}\right|\right)\right\} \\
& \leqq \\
& \sum_{i=1}^{n}\left\{F\left(b_{1}, \cdots, b_{i-1}, x_{i}+\left|h_{i}\right|, b_{i+1}, \cdots, b_{n}\right)\right. \\
&\left.\quad-F\left(b_{1}, \cdots, b_{i-1}, x_{i}-\left|h_{i}\right|, b_{i+1}, \cdots, b_{n}\right)\right\}<\epsilon .
\end{aligned}
$$


The first sum is obtained by elementary addition and subtraction, our functional symbols in the summand being subject to the convention that any argument with subscript outside the range 1 to $n$ which may seem to appear is understood to have been omitted. To see the next to the last inequality consider the typical difference in which the $i$ th coordinate is changed from $x_{i}+\left|h_{i}\right|$ to $x_{i}-\left|h_{i}\right|$. If $\alpha=\left(x_{1}\right.$ $\left.+\left|h_{1}\right|, x_{i}-\left|h_{i}\right|\right), \beta=\left(b_{1}, x_{i}+\left|h_{i}\right|\right), \nu=1, \eta=i, p=\left(x_{2}+\left|h_{2}\right|, \cdots\right.$, $\left.x_{i-1}+\left|h_{i-1}\right|, x_{i+1}-\left|h_{i+1}\right|, \cdots, x_{n}-\left|h_{n}\right|\right)$ then

$$
\Delta F_{1 i p}(\alpha, \beta) \geqq 0
$$

implies

$$
\begin{aligned}
F\left(x_{1}+\left|h_{1}\right|, \cdots,\right. & \left.x_{i}+\left|h_{i}\right|, \cdots, x_{n}-\left|h_{n}\right|\right) \\
& -F\left(x_{1}+\left|h_{1}\right|, \cdots, x_{i}-\left|h_{i}\right|, \cdots, x_{n}-\left|h_{n}\right|\right) \\
\leqq & F\left(b_{1}, x_{2}+\left|h_{2}\right|, \cdots, x_{i}+\left|h_{i}\right|, \cdots, x_{n}-\left|h_{n}\right|\right) \\
& \quad-F\left(b_{1}, x_{2}+\left|h_{2}\right|, \cdots, x_{i}-\left|h_{i}\right|, \cdots, x_{n}-\left|h_{n}\right|\right) .
\end{aligned}
$$

After $n-2$ applications of $\Delta \geqq 0$ we take $\alpha=\left(x_{i}-\left|h_{i}\right|, x_{n}-\left|h_{n}\right|\right)$, $\beta=\left(x_{i}+\left|h_{i}\right|, b_{n}\right), p=\left(b_{1}, \cdots, b_{i-1}, b_{i+1}, \cdots, b_{n-1}\right), \nu=i, \eta=n$. Since $\Delta F_{\text {imp }}(\alpha, \beta) \geqq 0$ we have

$$
\begin{aligned}
& F\left(b_{1}, \cdots, b_{i-1}, x_{i}+\left|h_{i}\right|, b_{i+1}, \cdots, x_{n}-\left|h_{n}\right|\right) \\
& -F\left(b_{1}, \cdots, b_{i-1}, x_{i}-\left|h_{i}\right|, b_{i+1}, \cdots, x_{n}-\left|h_{n}\right|\right) \\
& \leqq F\left(b_{1}, \cdots, b_{i-1}, x_{i}+\left|h_{i}\right|, b_{i+1}, \cdots, b_{n}\right) \\
& -F\left(b_{1}, \cdots, b_{i-1}, x_{i}-\left|h_{i}\right|, b_{i+1}, \cdots, b_{n}\right) .
\end{aligned}
$$

This procedure yields the desired inequality and completes the proof of the theorem.

THEOREM 2. Let $\left\{F_{q}\left(x_{1}, x_{2}, \cdots, x_{n}\right)\right\}$ be a sequence of monotone functions on $S$, and let $A$ be a positive number such that $\mid F_{q}\left(x_{1}, x_{2}, \cdots\right.$, $\left.x_{n}\right) \mid \leqq A$ for $q=0,1,2, \cdots$. There exists a sequence of integers

$$
q_{0}<q_{1}<q_{2}<\cdots
$$

and a monotone function $F\left(x_{1}, x_{2}, \cdots, x_{n}\right)$ bounded by $\pm A$ such that

$$
\lim _{i \rightarrow \infty} F_{q_{i}}\left(x_{1}, x_{2}, \cdots, x_{n}\right)=F\left(x_{1}, x_{2}, \cdots, x_{n}\right)
$$

on $S$.

In the proof we shall have use for the following selection principle $\left[6\right.$, p. 26]. Let the real constants $a_{m, q}$ be such that $\left|a_{m, q}\right| \leqq A$, for $m, q=0,1,2, \cdots$. Then there exists a sequence of positive integers

$$
q_{0}<q_{1}<q_{2}<\cdots
$$


and a set of reals $a_{0}, a_{1}, a_{2}, \cdots$ such that

$$
\lim _{i \rightarrow \infty} a_{m, q_{i}}=a_{m}, \quad \text { for } m=0,1,2, \cdots .
$$

Arrange the rational points (i.e. points with all coordinates rational) of $S$ in a sequence

$$
\left\{x^{m}\right\}=\left\{\left(x_{1}^{m}, x_{2}^{m}, \cdots, x_{n}^{m}\right)\right\}
$$

and consider the sequence

$$
a_{m, q}=F_{q}\left(x^{m}\right)=F_{q}\left(x_{1}^{m}, \cdots, x_{n}^{m}\right), \quad m, q=0,1,2, \cdots .
$$

By the selection principle given above we secure a sequence of integers

$$
q_{0,0}<q_{0,1}<q_{0,2}<\cdots
$$

such that

$$
\lim _{i \rightarrow \infty} F_{q_{0, i}}\left(x^{m}\right)=F\left(x^{m}\right)
$$

Let

$$
\limsup _{i \rightarrow \infty} F_{q_{0, i}}(x)=F^{*}(x), \quad \lim _{i \rightarrow \infty} \inf F_{q_{0, i}}(x)=F_{*}(x),
$$

for $x=\left(x_{1}, \cdots, x_{n}\right) \in S$. Clearly,

$$
F\left(x^{m}\right)=F^{*}\left(x^{m}\right)=F_{*}\left(x^{m}\right), \quad m=0,1,2, \cdots .
$$

One verifies that the functions $F^{*}(x)$ and $F_{*}(x)$ are monotone on $S$. Let $y=\left(y_{1}, \cdots, y_{n}\right) \in S$ be an irrational point which is a common point of continuity of $F^{*}$ and $F_{*}$. Then $F^{*}(y)=F_{*}(y)$ since $y$ can be approximated by rational points. We define

$$
F(y)=F^{*}(y)=F_{*}(y) \text {. }
$$

At this stage of the proof we have defined $F$ at all rational points and at all common points of continuity of $F^{*}$ and $F_{*}$.

The remainder of the proof is by induction on $n$. The theorem has been proved [6, p. 27] for $n=1$ and we now assume the conclusion holds for $n-1$.

By Theorem 1, the discontinuities of $F^{*}$ and $F_{*}$ lie on a set $R$ consisting of the union of sets of the form $x_{i}=\alpha_{i}^{j}$. Let $\Gamma_{i}^{j}$ be all points of $x_{i}=\alpha_{i}^{j}$ and order the sets $\Gamma_{i}^{j}$ by the agreement that $\Gamma_{r}^{s}$ precedes $\Gamma_{t}^{u}$ if $s<u$ or if $s=u$ and $r<t$. Denote the resulting sequence by $\left\{\Gamma_{o}\right\}$.

$\Gamma_{1}$ is an $(n-1)$-cell hence, by our inductive hypothesis, there is a subsequence 


$$
q_{1,0}<q_{1,1}<q_{1,2}<\cdots
$$

of (1) such that $\lim _{i \rightarrow \infty} F_{q_{1, i}}(x)$ converges on $\Gamma_{1}$ and clearly this function will agree with $F(x)$ wherever it has been defined.

Now, using induction on $g$, for each $g$ there is a sequence

$$
q_{g, 0}<q_{g, 1}<q_{0,2}<\cdots,
$$

a subsequence of $(1),(2), \cdots,(g)$, such that

$$
\lim _{1 \rightarrow \infty} F_{q_{g, i}}(x)
$$

exists on $\Gamma_{1}, \Gamma_{2}, \cdots, \Gamma_{g}$ and at all points where $F(x)$ was defined it will agree with $F(x)$.

Finally $\lim _{i \rightarrow \infty} F_{q_{i, i}}(x)$ exists at all points of $S$ and is monotone in $S$. The proof of the theorem is complete upon observing that we may take $q_{i} \equiv q_{i, i}$

If $x^{1}$ and $x^{2}$ are points of $S$, we write $x^{1} \prec \prec x^{2}$ if each coordinate of $x^{1}$ is less than the corresponding coordinate of $x^{2}$ and $x^{1}<x^{2}$ if no coordinate of $x^{1}$ is greater than the corresponding coordinate of $x^{2}$.

For an arbitrary point $v$ of $S$ let $[a, v)$ denote the interval $\{x: x \in S$, $x \prec \prec v\}$ and let $[a, v]$ denote the interval $\{x: x \in S, x \prec v\}$. A Lebesgue measurable set of $S$ which is the union of intervals of the type $[a, v)$ and (or) $[a, v]$ will be termed a lower layer. The boundary of a lower layer will be termed a monotone graph. In particular, if $F(x)$, $x=\left(x_{1}, x_{2}, \cdots, x_{n}\right)$, is monotone in its separate variables and $r$ is a real constant, then the set $\{x: x \in S, F(x)<r\}$ is a lower layer.

Let $M$ denote the class of all extended real valued functions $F(x)$, $x=\left(x_{1}, x_{2}, \cdots, x_{n}\right),-\infty \leqq F(x) \leqq+\infty$, defined on $S$, monotone nondecreasing in each of their $n$ separate variables and having nonnegative second difference when the four points of the second difference determined by $\alpha, \beta, p$ are in the complement of the closure of the set on which the function is $+\infty$ or $-\infty$.

For such extended monotone functions one is able to prove the following theorem using Theorem 2 as a lemma.

ThEOREM 3. Let $\left\{F_{q}(x)\right\}$ be a sequence of elements of $M$, then there exists a sequence of integers

$$
q_{0}<q_{1}<q_{2}<\cdots
$$

and at least one function $F(x) \in M$ such that $\lim _{i \rightarrow \infty} F_{q_{i}}(x)=F(x)$ except on at most two monotone graphs.

The two possible exceptional monotone graphs are, respectively, the boundaries of lower layers where $F_{*}=F^{*}=-\infty$ and where 
$F_{*} \leqq F^{*}<\infty$; here $F_{*}$ and $F^{*}$ are, respectively, lower and upper limits of a subsequence which converges at each rational point of $S$. The proof of Theorem 3 uses Theorem 2, but does not require explicitly a theorem describing the discontinuities of a function of $M$. However, the following analog of Theorem 1 is easily established.

TheOREM 4. If $F(x) \in M$, there is a set $R$ consisting of points of a countable collection of $(n-1)$-spaces $x_{i}=\alpha_{i}^{j}, \quad i=1,2, \cdots, n$; $j=1,2, \cdots$, together with at most two monotone graphs such that $F(x)$ is continuous at all points of $S-R$.

It is understood in the statement of Theorem 4 that if $F(m)$ $=+\infty(-\infty)$ and $F(x)$ is $+\infty(-\infty)$ in a neighborhood of $m$, then $F(x)$ is continuous at $m$.

3. Functions monotone in their separate variables. The first theorem stated in this section concerns a function $F(x, y)$ of two variables and is given by Hobson [4, p. 390] for functions monotone in their separate variables (Hobson uses the term "quasi-monotone" for the term "monotone" of this paper). That Hobson's theorem is false ${ }^{2}$ can be seen from the example $f(x, y)=0, x+y<0 ; f(x, y)=1, x+y \geqq 0$. Hobson's proof is valid in quadrants I and III but fails in II and IV.

THEOREM 5. Let $F(x, y)$ be monotone in a plane region with $(a, b)$ as an interior point. Consider the "quadrants" I: $x>a, y>b$; II: $x<a$, $y>b$; III: $x<a, y<b$; IV: $x>a, y<b$ about the point $(a, b)$. In any one of these quadrants $\lim _{x \rightarrow a, y \rightarrow b} F(x, y)=\lim _{x \rightarrow a} \lim _{y \rightarrow b} F(x, y)=\lim _{y \rightarrow b}$ $\lim _{x \rightarrow a} F(x, y)$ where in each case the approach to $a, b$ and $(a, b)$ is confined to the given quadrant.

We have no need for Theorem 5 in this paper and omit its proof. Since Hobson's proof is valid in quadrants I and III, the limit exists for an approach to $(a, b)$ within either of these quadrants, even if $F$ is only monotone in $x$ and $y$ separately and fails to have a second difference of constant sign. It is of interest to observe further that if the limits from quadrants I and III are identical, $F$ is actually continuous at $(a, b)$. Indeed, we have the following result, in which the continuity hypotheses on $F$ at $(a, b)$ are only apparently weaker than one requiring the limits from within I and III to coincide.

TheOREM 6. Let $F(x), x=\left(x_{1}, x_{2}, \cdots, x_{n}\right), x \in S$, be monotone nondecreasing in each variable $x_{i}$. Let $m=\left(m_{1}, \cdots, m_{n}\right)$ be a point of $S$ and denote by I all $x \in S$ such that $x_{i}>m_{i}, i=1,2, \cdots, n$, and by III all $x \in S$ such that $x_{i}<m_{i}, i=1,2, \cdots, n$. If for each $\epsilon>0$ there exists $\alpha=\left(\alpha_{1}, \alpha_{2}, \cdots, \alpha_{n}\right) \in \mathrm{I}$ and $\beta=\left(\beta_{1}, \beta_{2}, \cdots, \beta_{n}\right) \in \mathrm{III}$ for which $F(\alpha)$ $-F(\beta)<\epsilon$ then $F(x)$ is continuous at $m$. 
Proof. Given $\epsilon>0$ let $x$ be any point of $S$ such that $\alpha_{i} \leqq x_{i} \leqq \beta_{i}$, $i=1,2, \cdots, n$, then $|F(x)-F(m)| \leqq F(\alpha)-F(\beta)<\epsilon$ and the proof is complete.

We observe that the condition: corresponding to each $\epsilon>0$, are $\alpha, \beta$ in I and III, respectively, such that $F(\alpha)-F(\beta)<\epsilon$, is equivalent, for example, to the condition: there exists a line through $m$ lying in I and III on which $F(x)$ is continuous at $m$.

In the final two theorems of the paper we describe the discontinuities of functions defined on $S$ and monotone in their separate variables and show that a Helly theorem similar to Theorem 2 is impossible for such functions.

TheOREM 7. If $F(x), x=\left(x_{1}, x_{2}, \cdots, x_{n}\right)$, is finite and monotone in its separate variables on $S$, then the discontinuities of $F(x)$ lie on a countable set of monotone graphs of $S$.

Proof. For each rational number $r$, let $\Gamma_{r}$ denote the monotone graph defined by the lower layer $C_{r}=\{x: x \in S, F(x)<r\}$. That is, $\Gamma_{r}$ is the boundary of $C_{r}$. We shall show that any point of discontinuity of $F(x)$ is on $\Gamma_{r}$ for some rational $r$.

If $m \in S$ is a point of discontinuity of $F(x)$, by Theorem 6,

$$
\lim _{x \rightarrow m, x \in \mathrm{I}} F(x) \neq \lim _{x \rightarrow m, x \in \mathrm{III}} F(x) .
$$

If $r$ is any rational number between these limits, then $m \in \Gamma_{r}$ and the theorem is proved.

It is easily seen that the preceding theorem holds when $F(x)$ is permitted to take on the values $+\infty$ and $-\infty$.

THEOREM 8. There exists a uniformly bounded infinite sequence of functions monotone nondecreasing in their two variables on a rectangle $S$ such that no infinite subsequence of the sequence converges on $S$ to a single valued function.

To prove Theorem 8 it suffices to take the unit square as $S$ and to define $F_{n}(x, y) \equiv 0$ for $x+y<1, F_{n}(x, y) \equiv 1$ for $x+y>1, F_{n}(x, 1-x)$ $\equiv \phi_{n}(x), n=1,2, \cdots$, where $\phi_{n}(x)$ is a sequence of functions defined on $[0,1]$, bounded between 0 and 1 , from which no everywhere convergent subsequence can be extracted.

\section{BIBLIOGRAPHY}

1. S. Bochner, Monotone Funktionen, Stieltjessche Integrale und harmonische Analyse, Math. Ann. vol. 108 (1933) pp. 378-410.

2. H. Cramér, Mathematical methods of statistics, Princeton, Princeton University Press, 1946. 
3. E. Helly, Über lineare funktional operationen, Sitzungsberichte der Mathematisch-Naturwissenschaftlichen Klasse der Kaiserlichen Akademie der Wissenschaften vol. 121 (1912) pp. 265-297.

4. E. W. Hobson, The theory of functions of a real variable and the theory of Fourier's series, 2d ed., Cambridge, Cambridge University Press, 1921.

5. J. A. Shohat and J. D. Tamarkin, The problem of moments, Mathematical Surveys no. 14, New York, 1943.

6. D. V. Widder, The Laplace transform, Princeton University Press, 1941.

7. W. H. Young, On multiple integrals, Proc. Roy. Soc. London Ser. A vol. 93 (1916) pp. 28-41.

UNIVERSITY OF MisSOURI

\section{ABELIAN RINGS AND SPECTRA OF OPERATORS ON $l_{p}$}

\section{G. L. KRABBE}

1. Introduction. Let $l_{p}$ denote the set of all sequences $c=\left\{c_{n}\right\}$ such that $\|c\|_{p}=\left(\sum_{n=-\infty}^{\infty}\left|c_{n}\right|^{p}\right)^{1 / p}<\infty$. If $a$ and $c$ are sequences, the convolution $a * c$ is defined as the sequence $\left\{b_{n}\right\}$ such that

$$
[a * c]_{n}=b_{n}=\sum_{\nu=-\infty}^{\infty} a_{n-\nu} c_{\nu} \quad(n=0, \pm 1, \pm 2, \pm 3, \cdots) .
$$

Suppose $A$ is a bounded and summable function on the interval $[-\pi, \pi]$, and denote by $\Phi A$ the sequence $\left\{a_{n}\right\}$ of Fourier coefficients of $A$. The "Laurent matrix" $\left(a_{n-v}\right)$ represents a transformation $A t_{p}$ defined for any $c$ in $l_{p}$ by $A \#_{p} c=(\Phi A) * c$. The following properties were proved for $p=2$ by O. Toeplitz [11] and F. Riesz [8, pp. 171175]:

(i) $A_{t_{p}}$ is a bounded operator on $l_{p}$,

(ii) if $B(\theta)=[A(\theta)]^{-1}$ defines a bounded function $B$, then the inverse $\left(A_{*}\right)^{-1}$ of $A_{*_{p}}$ exists, and $\left(A_{\#_{p}}\right)^{-1}=B_{\#_{p}}$,

(iii) if $A$ is continuous, then the spectrum $\sigma\left(A_{p}\right)$ of $A_{\sharp p}$ is the range $e^{1}$ of $A$.

Assume henceforth that $p>1$. This case was not considered by Toeplitz and Riesz; their results depend on the circumstance ${ }^{2}$ that

Presented to the Society April 15, 1955 under the titles Certain abelian rings of operators on $l_{p}$ and $O n$ the spectra of certain operators on $l_{p}$; received by the editors January 31, 1955 and, in revised form, July 25, 1955 and October 11, 1955.

1 The range of $A$ is the image $A([-\pi, \pi])$.

2 The introductions to $[3 ; 4]$ contain a concise account of these matters. 